\title{
GADD45aa as a Predictive Biomarker for Aeromonas Infection-Related Liver Cirrhosis
}

\author{
Hamid Sadeghi ${ }^{1} \cdot$ Nematollah Gheibi $^{2}$ Saeideh Gholamzadeh Khoei ${ }^{2,3}$ (D)
}

Accepted: 12 August 2021

๑) Springer Science+Business Media, LLC, part of Springer Nature 2021

Liver cirrhosis is recognized as the 11th leading cause of mortality in the world [1]. Cirrhosis is the last pathological outcome of different chronic liver disorders, and newer research has established that fibrosis is the starter of cirrhosis [2-4]. Many types of factors are involved in the beginning and development of liver fibrosis and cirrhosis [3]. The most common causes in the liver cirrhosis progression, especially in western countries, include alcoholism, nonalcoholic fatty liver disease, and chronic hepatitis $\mathrm{C}$ virus infection [5]. In addition, Gram-negative bacteria such as Escherichia coli, Klebsiella sp., and Aeromonas sp., can be important origins of bacteremia in patients with liver cirrhosis [6,7].

In recent years, several effective measures have been developed in order to address the development of antifibrotic methods. Although elimination of causative factors of liver fibrosis will result in tissue scarring, it is troublous to treat advanced liver cirrhosis [8]. One approach to inhibit liver-related mortality is to detect and inhibit the progression of bacteremia [9]. It is interesting that cirrhosis patients with more acute hepatic dysfunction are mostly considered to be linked with a higher rate of inception of bacteremia and the more severe the bacteremia, the worse prognosis [10]. Some past studies provided evidence that Aeromonas sp. infections promote liver fibrosis and cirrhosis by the acquisition of liver tissue [7, 11]; however, few studies have been carried out in this area.

Saeideh Gholamzadeh Khoei

s.gholamzade@yahoo.com

1 Department of Microbiology and Virology, School of Medicine, Zanjan University of Medical Sciences, Zanjan, Iran

2 Cellular and Molecular Research Center, Research Institute for Prevention of Non-Communicable Diseases, Qazvin University of Medical Sciences, Qazvin, Iran

3 Department of Medical Biotechnology, School of Advanced Medical Sciences and Technologies, Hamadan University of Medical Sciences, Hamadan, Iran
Aeromonas sp. are oxidase-producing Gram-negative that have been implicated in a variety of human diseases. They are found overall in the natural world [12]. This genus includes 36 species that are isolated from aquatic environments, animals, foods, and diverse infectious in humans. [13]. Various species of Aeromonas are addressed as emerging pathogens on account of causing a broad spectrum of disease in humans, such as wound infections, gastroenteritis, and septicemia/bacteremia, infecting immunocompetent and immunocompromised [14]. Study after study has shown that $96.5 \%$ of the strains related to clinical cases were recognized as one of only four species: Aeromonas caviae (29.9\%), Aeromonas dhakensis (26.3\%), Aeromonas veronii (24.8\%), and Aeromonas hydrophila (15.5\%) [15]. Aeromonas bacteremia is infrequent in healthy people but is often detected in patients with hematological malignancy, hepatobiliary infection, and decompensated liver cirrhosis [13]. It has been distinguished that liver cirrhosis patients are susceptible to aggressive Aeromonas infections [16]. Therefore, identifying the molecular biomarkers is urgently needed to underlie the Aeromonas infections in liver cirrhosis and develop novel diagnostic and prognostic agents for Aeromonas infections that will enhance the development of therapeutic strategies for cirrhosis patients.

GADD45 (growth arrest and DNA damage-inducible 45) genes including Gadd45aa and Gadd45ab have been implicated in signaling responses to genotoxic stress agents, cell cycle arrest, cell survival, DNA repair, apoptosis, and innate immune [17]. The induction of Gadd45 members occurs differentially and their functions usually overlap [18]. According to previously reported evidence, GADD45aa expression was downregulated in the liver but GADD45ab upregulated [19]. Also, in grass carp with Aeromonas hydrophila, the expression of both Gadd45aa and Gadd45ab were upregulated [19]. Therefore, the liver infected with Aeromonas sp. is expected to show high expression of Gadd45aa and Gadd45ab. Hence, it seems that, due to the low expression of Gadd45aa in the liver, this gene can be addressed as a 
biomarker in Aeromonas infection-related liver cirrhosis. It should be noted that the study of protein and mRNA has its own importance on account of the strict control levels in both transcription and translation, and it seems that the expression of them may not be the same and need to examine both. In the current study, we hypothesized that the investigation of GADD45aa gene expression, as a candidate biomarker, in the prognosis of Aeromonas infection-related liver cirrhosis can be effective and provide the best cure choice for treatment. Indeed, if Aeromonas infection is the cause of liver cirrhosis, the presence of infection can be detected by GADD45aa expression follow-up, and it can be treated before the fibrosis reaches cirrhosis. Clearly, more direct and in vivo evaluations are needed to characterize the role of GADD45 in Aeromonas sp. infection and liver cirrhosis.

Author Contribution The authors contributed to the research conception and design. All authors studied this draft and contributed and confirmed the final manuscript.

\section{Declarations}

Ethics Approval Consent to Participate Not applicable.

Consent for Publication All the authors approved the final manuscript to be published and agreed to be accountable for all aspects of the study.

Conflict of Interest The authors declare no competing interests.

\section{References}

1. Asrani SK, Devarbhavi H, Eaton J, Kamath PS. Burden of liver diseases in the world. J Hepatol. 2019;70(1):151-71.

2. Smith A, Baumgartner K, Bositis C. Cirrhosis: diagnosis and management. Am Fam Physician. 2019;100(12):759-70.

3. Zhou W-C, Zhang Q-B, Qiao L. Pathogenesis of liver cirrhosis. World J Gastroenterol: WJG. 2014;20(23):7312.

4. Mohagheghi S, Khajehahmadi Z, Nikeghbalian S, Alavian SM, Doosti-Irani A, Khodadadi I, et al. Changes in the distribution of etiologies of cirrhosis among patients referred for liver transplantation over 11 years in Iran. Eur J Gastroenterol Hepatol. 2020;32(7):844-50.
5. Innes HA, Hutchinson SJ, Barclay S, Cadzow E, Dillon JF, Fraser A, et al. Quantifying the fraction of cirrhosis attributable to alcohol among chronic hepatitis $\mathrm{C}$ virus patients: implications for treatment cost-effectiveness. Hepatology. 2013;57(2):451-60.

6. Caly WR, Strauss E. A prospective study of bacterial infections in patients with cirrhosis. J Hepatol. 1993;18(3):353-8.

7. Shizuma T, Tanaka C, Mori H, Fukuyama N. Investigation of bacteremia due to Aeromonas species and comparison with that due to enterobacteria in patients with liver cirrhosis. Gastroenterol Res Pract. 2011;2011.

8. Brenner DA. Reversibility of liver fibrosis. Gastroenterology \& hepatology. 2013;9(11):737.

9. Yan J, Li S, Li S. The role of the liver in sepsis. Int Rev Immunol. 2014;33(6):498-510.

10. Shizuma T, Obata H, Hashimoto E, Shiratori K. Relationship between bacteremia and severity of liver dysfunction in patients with liver cirrhosis. Acta Hepatologica Japonica. 2003;44(12):641-8.

11. Wu C-J, Lee H-C, Chang T-T, Chen C-Y, Lee N-Y, Chang C-M, et al. Aeromonas spontaneous bacterial peritonitis: a highly fatal infectious disease in patients with advanced liver cirrhosis. J Formos Med Assoc. 2009;108(4):293-300.

12. Mehta AK, Lyon GM. Infectious diseases in end-stage liver disease patients. Critical Care Nursing Clinics. 2010;22(3):291-307.

13. Janda JM, Abbott SL. The genus Aeromonas: taxonomy, pathogenicity, and infection. Clin Microbiol Rev. 2010;23(1):35-73.

14. Figueras M, Suarez-Franquet A, Chacon M, Soler L, Navarro M, Alejandre $\mathrm{C}$, et al. First record of the rare species Aeromonas culicicola from a drinking water supply. Appl Environ Microbiol. 2005;71(1):538-41.

15. Latif Eugenín FL. Aeromonas, un microorganismo ambiental de importancia en salud humana y animal: Universitat Rovira i Virgili. 2015.

16. Ko W-C, Lee H-C, Chuang Y-C, Liu C-C, Wu J-J. Clinical features and therapeutic implications of 104 episodes of monomicrobial Aeromonas bacteraemia. J Infect. 2000;40(3):267-73.

17. Cretu A, Sha X, Tront J, Hoffman B, Liebermann DA. Stress sensor Gadd45 genes as therapeutic targets in cancer. Cancer Therapy. 2009;7(A):268.

18. Balliet AG, Hollander MC, Fornace AJ Jr, Hoffman B, Liebermann DA. Comparative analysis of the genetic structure and chromosomal mapping of the murine Gadd45g/CR6 gene. DNA Cell Biol. 2003;22(7):457-68.

19. Fang Y, Xu XY, Shen Y, Li J. Molecular cloning and functional analysis of Growth arrest and DNA damage-inducible 45 aa and ab (Gadd45aa and Gadd45 ab) in Ctenopharyngodon idella. Fish Shellfish Immunol. 2018;77:187-93.

Publisher's Note Springer Nature remains neutral with regard to jurisdictional claims in published maps and institutional affiliations. 